\title{
Angle Closure Imaging: A Review
}

\author{
John Mark S. de Leon - Tin A. Tun • \\ Shamira A. Perera $\cdot$ Tin Aung
}

Published online: 23 March 2013

(C) Springer Science + Business Media New York 2013

\begin{abstract}
Primary angle closure glaucoma (PACG) is a major form of glaucoma. Early detection and proper management of PACG to reduce visual loss are intricately related to correctly assessing the anterior chamber angle (ACA). This review describes clinical assessment of the ACA by gonioscopy, as well as novel ACA imaging devices and discusses their advantages and limitations. Specifically, we review ultrasound biomicroscopy, anterior segment optical coherence tomography, and a method similar to goniophotography using the EyeCam ${ }^{\mathrm{TM}}$, all of which are used to assess the ACA directly. In addition, we discuss surrogate approaches to measuring angle configuration by Scheimpflug photography and the SPAC scanning peripheral anterior chamber depth analyzer.
\end{abstract}

Keywords Anterior chamber angle imaging - Anterior segment optical coherence tomography - EyeCam . Gonioscopy · Ultrasound biomicroscopy

\section{Introduction}

Primary angle closure glaucoma (PACG) is one of the leading causes of blindness worldwide [1-3]. The primary risk factor is iridotrabecular contact that may impair aqueous humor drainage from the anterior chamber caused

J. M. S. de Leon · T. A. Tun · S. A. Perera · T. Aung ( $₫)$ Singapore Eye Research Institute, Singapore National Eye Center, 11 Third Hospital Avenue,

Singapore 168751, Singapore

e-mail: aung_tin@yahoo.co.uk

T. Aung

Yong Loo Lin School of Medicine, National University of Singapore, Singapore, Singapore by the mechanical obstruction of the functional trabecular meshwork (TM) by the peripheral iris and/or by abnormalities at the ultrastructural level of the TM caused by the prolonged friction/apposition of the iris against the angle wall [4]. Detection of appositional (nonpermanent) angle closure is an essential step in PACG blindness prevention as interventions such as laser peripheral iridotomy (LPI) may halt the angle closure process, preventing the development of glaucomatous optic neuropathy.

\section{Gonioscopy}

Dynamic dark room indentation indirect gonioscopy is the current gold standard for assessing the ocular anterior chamber angle (ACA) structures. Gonioscopy does not require expensive or sophisticated equipment beyond a gonioscopy lens and slit lamp. Aside from clinically differentiating between open and closed angles, indentation gonioscopy permits differentiation between appositional and synechial (permanent) closure. Gonioscopy, however, requires a fair amount of examiner skill, and the findings are subjective and more qualitative than quantitative. Gonioscopy findings can be affected by room lighting, globe pressure, and gaze direction, and the examiner is unable to visualize the structures posterior to the iris, such as the ciliary body and ciliary processes. Performing a quick, reliable, and reproducible gonioscopic assessment of the ACA may represent a major challenge in daily clinical practice.

Various angle imaging devices have been developed to aid in the objective and reproducible assessment of the ACA. Ultrasound biomicroscopy (UBM), Visante ${ }^{\mathrm{TM}}$ anterior segment optical coherence tomography (AS-OCT) (Carl-Zeiss Meditec, Dublin, CA, USA), spectral-domain 
optical coherence tomography (SD-OCT) $\left[\mathrm{RTVue}^{\circledR}\right.$ (Optovue, Fremont, CA, USA), Cirrus ${ }^{\mathrm{TM}}$ high-definition optical coherence tomography (HD-OCT) 4.0 (Carl Zeiss Meditec, Dublin, CA, USA), Spectralis ${ }^{\circledR}$ anterior segment module (Heidelberg Engineering, Dossenheim, Germany)], swept-source optical coherence tomography (CASIA $^{\mathrm{TM}}$ SS-1000; Tomey, Nagoya, Japan), EyeCam ${ }^{\mathrm{TM}}$ (Clarity Medical Systems, Pleasanton, CA, USA), Scheimpflug photography (Pentacam ${ }^{\circledR}$; Oculus Optikgeräte, Wetzlar, Germany), and the SPAC scanning peripheral anterior chamber depth analyzer (Takagi Seiko, Nagano-ken, Japan) all provide insight into the configuration of the ACA. These devices provide more objective measures of the ACA, which may allow more accurate determination of risk related to angle closure glaucoma.

This is a review of these devices concentrating on their utility for ACA assessment. The underlying principle, image acquisition technique, comparison with the reference standard, angle parameters measured, how they compare with each other, and strengths and limitations of each of them will be detailed.

\section{Ultrasound Biomicroscopy}

UBM is a technique developed in the 1990s that uses electric signals converted to high-frequency $(35-100-\mathrm{MHz})$ sound waves (by a radiofrequency signal generator coupled to a piezoelectric transducer) that are transmitted to the eye via a coupling solution held in a cup reservoir or within the end of a probe on which the transducer is mounted. These sound waves travel at differing velocities through the eye as they encounter tissues of different acoustic impedance and are reflected at differing time intervals. UBM scans at a rate of eight frames per second and produces a $5 \mathrm{~mm} \times 5 \mathrm{~mm}$ field with 256 image lines. It provides an axial and lateral resolution of 25 and $50 \mu \mathrm{m}$, respectively, and a tissue depth penetration of 5-7 $\mathrm{mm}$. A computer system collates and magnifies these reflected sound waves, providing high-resolution real-time or static in vivo crosssectional B-scan images.

For proper ACA evaluation, UBM images must be acquired perpendicular to the structures of interest. The double arc on the cornea is one of the findings correlated with a properly acquired image. The scleral spur (SS) is the constant identifiable landmark and is the key to analyzing angle disease [5]. It can be identified in the area where the radiopaque scleral shadow merges with the relatively radiolucent shadow of the cornea.

UBM can show the extent of closure and the depth of the anterior and posterior chambers, and can be used to investigate other mechanisms behind angle closure, which may include lenticular causes, anterior rotation of the ciliary body in plateau iris, iridociliary masses, and choroidal effusions [6-8].

The following are some of the important ACA parameters that can be evaluated by UBM (Fig. 1):

1. Trabecular-iris angle (TIA) The angle measured with the apex in the iris recess and the arms of the angle passing through a point on the TM $500 \mu \mathrm{m}$ from the SS and the point on the iris perpendicularly opposite.

2. Angle opening distance (AOD) AOD 250/500 is calculated from the corneal endothelium to the anterior iris and is measured at $250 \mu \mathrm{m}$ (at the level of the posterior TM, AOD 250) or $500 \mu \mathrm{m}$ (at the level of the anterior Schwalbe's line, AOD 500) from the SS.

3. Angle recess area (ARA) The area bordered by the anterior iris surface, corneal endothelium, and a line perpendicular to the corneal endothelium drawn to the iris surface from a point 500 or $750 \mu \mathrm{m}$ anterior to the SS. Instead of the iris being treated like a straight line as for the AOD, for the ARA irregularities of the iris contour are taken into account [9].

4. Trabecular-iris space area (TISA) The TISA at 500 or $750 \mu \mathrm{m}$ is a trapezoidal area limited anteriorly by AOD 500 or AOD 750, respectively, posteriorly by a line drawn from the SS perpendicular to the plane of the inner scleral wall to the opposing iris, superiorly by the inner corneoscleral wall, and inferiorly by the iris surface. Radhakrishnan et al. [10] first described the TISA to better define the filtering area compared with the ARA, excluding the nonfiltering region behind the SS.

A study of several UBM parameters [11] found high intraobserver reproducibility, but interobserver reproducibility differed considerably, and was affected by subjective interpretation of visualized anatomic landmarks (e.g., SS). UBM studies compared with gonioscopy have found a high agreement when both are performed in a completely dark room [12,13]. Gonioscopy occasionally resulted in an overestimation of the angle width as compared with UBM measurements in eyes with occludable angles [13], but angle dimensions measured by UBM correlated significantly with those measured by gonioscopy in general (even though the UBM results were more reproducible) [14].

The eye cup, commonly used in UBM imaging, introduces discomfort, the need for a supine position, risk of mechanical corneal abrasion and infection, and the possibility of angle distortion caused by inadvertent indentation [5]. However, recent UBM models with an eye cup integrated in the probe allow a sitting position. Earlier models are limited in their ability to provide images in only one eye quadrant at a time, but newer models acquire images of 
Fig. 1 Trabecular-iris angle (TIA) [yellow angle], angle opening distance $(A O D)$, angle recess area $(A R A)[$ yellow border], trabecular-iris space area (TISA) [red cross-hatching], $S S$-scleral spur, $C B$-ciliary body (Color figure online)

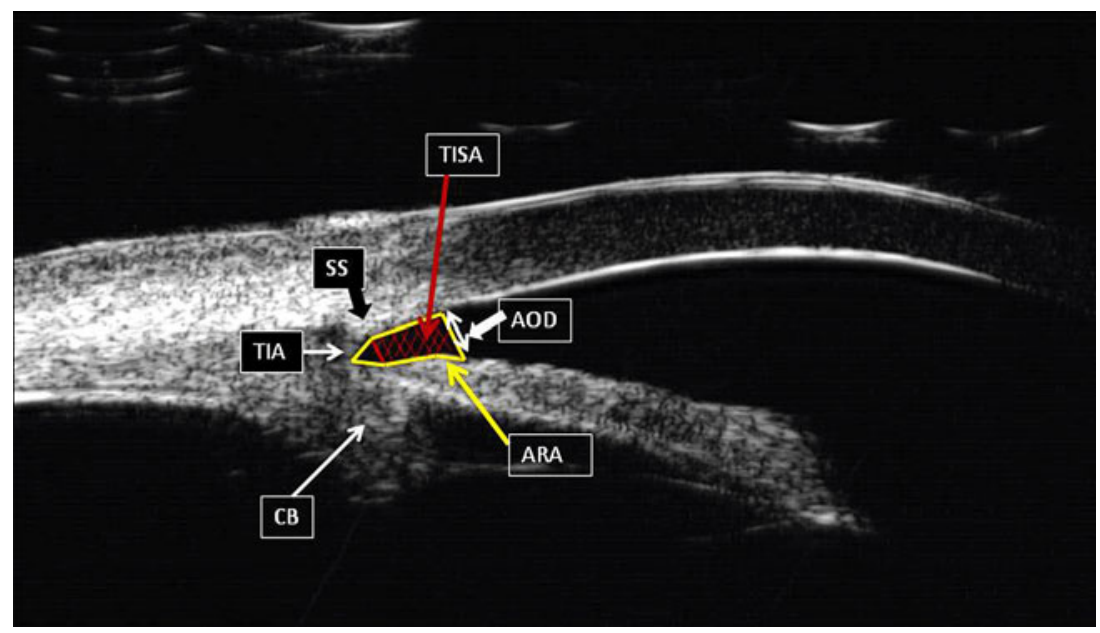

$180^{\circ}$ of the angle in a single frame. Optimal UBM imaging requires a skilled operator and a cooperative subject. Advantages of UBM include detailed imaging beyond the iris as deep as the pars plana, ciliary body, lens zonules, and anterior choroid. UBM can penetrate through poor ocular media (e.g., corneal edema) unlike light-based ACA imaging devices.

\section{Anterior Segment Optical Coherence Tomography}

AS-OCT uses low-coherence interferometry of light with a wavelength of $1,310 \mathrm{~nm}$ (vs $800 \mathrm{~nm}$ for posterior segment imaging) to provide increased penetration through lightscattering ocular structures, such as the sclera and iris, and thus improves visualization of anterior segment structures. AS-OCT produces noncontact in vivo cross-sectional images of tissues to obtain the reflectivity profile of the structures and allows their objective and quantitative evaluation. The light used by AS-OCT systems cannot image the anterior segment through the eyelids so the superior and inferior eyelids must be gently moved out of the way before scans of the superior and inferior ACA, respectively, are obtained.

The patient sits upright and image acquisition is faster than in UBM, which reduces motion artifacts and the examination time. After acquisition, scanned images are processed (dewarped) by custom software which compensates for the index of refraction transition of the air-tear interface and the different group indices in air, cornea, and aqueous humor to correct the physical dimensions of ocular images [15].

ACA images can be evaluated qualitatively by subjectively determining the open or closed angle status and quantitatively by objectively measuring ACA parameters. Both analyses are dependent on the recognition of cross-sectional anatomical landmarks since the TM, the anatomical site of aqueous drainage, cannot be visualized directly. A commonly used anatomical landmark is the SS, which is usually located on the widest part of the cross section of the sclera, appearing as an inward protrusion where some of the fibers of the longitudinal bundle of the ciliary muscle attach. It is an anatomical landmark that reveals the relative location of the TM, which is located approximately $250-500 \mu \mathrm{m}$ anterior to the SS along the angle wall [16].

In qualitative analysis, a closed ACA is defined as the presence of contact between the iris and angle wall anterior to the level of the SS (Figs. 2, 3). In quantitative analysis, AS-OCT with the help of customized software is capable of measuring novel angle parameters, some of which are similar to the UBM parameters already described. Again just like UBM, the precise identification of the SS is critical in the proper measurement of some of these parameters [16], which include the AOD (either 500 or $750 \mu \mathrm{m}$ ), ARA, and TISA.

Sakata et al. [17, 18] observed that the SS could not be identified in almost $30 \%$ of Visante $^{\mathrm{TM}}$ (model 1000, software version 1.0) AS-OCT images and that it was more difficult to identify the SS in eyes with narrow angles and in images obtained in the superior and inferior quadrants (which usually had narrower angles). Despite this, almost $90 \%$ of the AS-OCT images could still be qualitatively graded by glaucoma specialists as having either an open or a closed ACA [19, 20]. This was possible by relying on other morphological landmarks, such as iris insertion and the peripheral iris profile. The difficulty in determining the location of the SS may hinder quantitative analysis of ACA parameters in a substantial proportion of subjects [18], thereby influencing the utility of novel anterior segment parameters that seem to improve the diagnostic performance of ACA imaging devices in detecting eyes at risk of angle closure. As technology advances, updates to current software and hardware may improve image resolution, 
Fig. 2 Anterior segment optical coherence tomography image of open angles. The arrows point to the location of the scleral spur

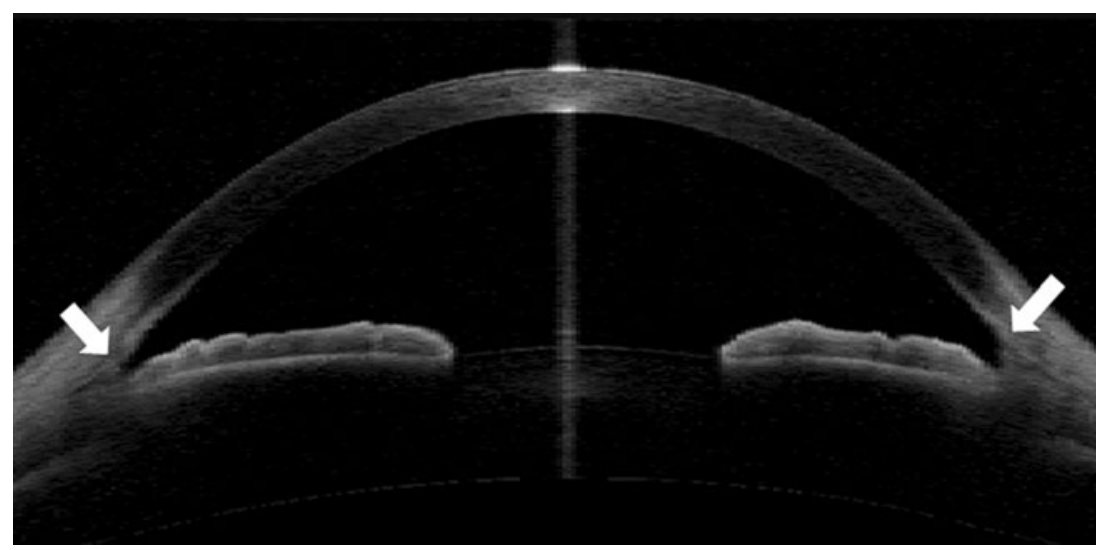

Fig. 3 Anterior segment optical coherence tomography image of closed angles. The arrows point to the location of the scleral spur

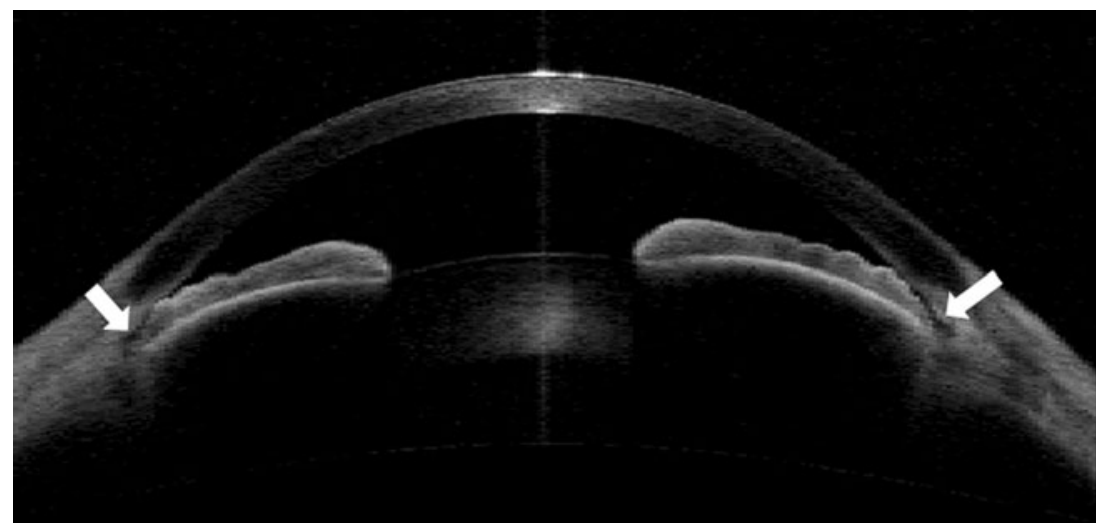

enhancing SS detection and possibly new anatomical reference points.

Console et al. [19] evaluated the reproducibility of the Visante $^{\mathrm{TM}}$ in eyes with open or narrow ACAs using customized software (Zhongshan Angle Assessment Program; Zhongshan Ophthalmic Center, Guangzhou, China). They observed that reproducibility was worse in eyes with a narrow ACA and that determination of the location of the SS by different examiners was an important source of variability.

Studies comparing AS-OCT and gonioscopy found the Visante $^{\mathrm{TM}}$ detected more closed angles than gonioscopy, particularly in the superior and inferior angle quadrants $[17,20]$. With use of the definition of one or more quadrants of TM nonvisibility as determined by gonioscopy, the sensitivity and specificity of the Visante ${ }^{\mathrm{TM}}$ to identify angle closure were 98 and $55.4 \%$, respectively [20]. This could be due to inadvertent pressure on the globe and too much light exposure during gonioscopy, which may widen the angle. Another reason could be different definitions of a closed angle. For gonioscopy, angle closure was defined as apposition between the iris and the posterior TM, whereas for the Visante ${ }^{\mathrm{TM}}$ it was any contact between the iris and the angle structures anterior to the SS.

Dada et al. [21] evaluated 63 subjects with normal eyes (open angles), and observed good correlation between
AS-OCT and UBM measurements obtained in the nasal and temporal quadrants. However, comparison between these methods in eyes with narrow angles remains to be evaluated. Radhakrishnan et al. [10] compared the quantitative measurements provided by a UBM system and a Visante $^{\mathrm{TM}}$ prototype in a small sample of 17 normal subjects and seven narrow ACA subjects as determined by gonioscopy. They observed that both methods had similar discriminatory power to detect eyes with narrow ACA. They also observed that there were significant differences between some ACA parameters (ARA 500 and $750 \mu \mathrm{m}$, TISA $750 \mu \mathrm{m}$ ) measured by the two devices, as UBM tended to show smaller measurements. There are only a few studies comparing UBM and AS-OCT, and they have a small sample size, selective inclusion/exclusion criteria, and/or only evaluate eyes with open ACA.

The advantages of AS-OCT include ease of operation, rapid image acquisition, and absence of contact with the eye, which eliminates discomfort and inadvertent globe compression. This is especially useful in trauma and postoperative eyes. The incorporation of automated analysis software allows rapid measurements of the various novel anterior segment parameters as previously mentioned, including corneal thickness and anterior chamber depth. The disadvantages include the inability to distinctly 
detect and measure structures posterior to the iris as well as peripheral anterior synechiae. Software analysis programs require manual SS localization, which may require observer skill, especially in closed angles or where there is a smooth transition from cornea to sclera. Although gonioscopy allows indentation and dynamic visualization of the entire angle quadrant, AS-OCT images should only be interpreted for specific cross sections of the ACAs scanned. The high cost of the devices used may be a limiting factor for their use in routine clinical setup or for screening purposes.

\section{Spectral-Domain Optical Coherence Tomography}

The software of three commercial posterior segment SD-OCT devices-RTVue ${ }^{\circledR}$ (Optovue, Fremont, CA, USA), Cirrus ${ }^{\mathrm{TM}}$ HD-OCT 4.0 (Carl Zeiss Meditec, Dublin, CA, USA), and Spectralis ${ }^{\circledR}$ (Heidelberg Engineering, Dossenheim, Germany) — was recently upgraded to include ACA imaging. The devices differ from those used in timedomain optical coherence tomography (TD-OCT) by using shorter wavelengths $(830 \mathrm{~nm})$, have fixed reference mirrors, allowing higher scanning speed (26,000 A-scans per second), take more images in a single pass, [22], and have higher axial $(5 \mu \mathrm{m})$ and transverse $(15 \mu \mathrm{m})$ resolution.

In a study [23] comparing the Cirrus ${ }^{\mathrm{TM}}$ and the RTVue ${ }^{\circledR}$ SD-OCT devices, it was more difficult to determine angle closure status with the Cirrus ${ }^{\mathrm{TM}}$ device than with RTVue ${ }^{\circledR}$ device (angle closure status was indeterminable in 14.5 and $50.7 \%$ of scans, respectively); however, there was fair agreement between both devices. Wylegala et al. [24] compared the RTVue ${ }^{\circledR}$ and Visante ${ }^{\mathrm{TM}}$ devices for measurement of TIA and AOD in 30 normal subjects, and did not find any significant differences between the instruments. Compared with the TD-OCT device, the SD-OCT device produces more detailed cross-sectional images of important structures (e.g.,TM and Schlemm's canal) [24, 25] (Figs. 4, 5).

A disadvantage of SD-OCT is that current software does not adjust for refraction at the air-cornea and corneaaqueous humor interfaces (dewarping). Imaging should be performed in a direction perpendicular to the limbus to minimize the effect of image distortion. The shorter wavelength of SD-OCT reduces the depth of penetration, making it less useful for the iris and more posterior areas, and in its current format, there are no wide-field optics for SD-OCT that allow the viewing of the entire anterior chamber in a single image as with TD-OCT [25].

Swept-Source Optical Coherence Tomography $\left(\right.$ CASIA $^{\mathrm{TM}}$ )

SS-OCT (CASIA $^{\text {TM}}$; Tomey, Nagoya, Japan) is a form of SD-OCT but instead of using a spectrometer (as used in other SD-OCT devices), it uses a monochromatic tunable fast scanning laser source and a photodetector to detect wavelength-resolved interference signal [26, 27]. The wavelength of the swept-source laser is $1,310 \mathrm{~nm}$ and the scan dimensions (low resolution) are $16 \mathrm{~mm}$ (width) $\times 16 \mathrm{~mm}$ (length) $\times 8 \mathrm{~mm}$ (depth). With a scan speed of 30,000 A-scans per second, the low-resolution mode can collect $128 \mathrm{~B}$-scans in $2.4 \mathrm{~s}$, and $64 \mathrm{~B}$-scans can be collected in $1.2 \mathrm{~s}$ with the high-resolution mode across the entire anterior chamber. A three-dimensional $360^{\circ}$ photograph-like view of the iris and the ACA can be reconstructed from individual image frames in the lowresolution mode, and the SS and Schwalbe's line can be visualized in the high-resolution mode (Fig. 6).

SS-OCT provides reproducible measurements of AOD, TISA, and TIA in different quadrants, suggesting that it is useful for measuring the angle for risk assessment of angle closure and for evaluating longitudinal changes before and after therapeutic interventions in patients with angle closure [28]. In addition, the interobserver and intraobserver agreement of the iridotrabecular contact index, a measure of the extent of angle closure using SS-OCT, is good [29•].

Another advantage is the three-dimensional view of the angle that may enable patients to better understand their condition prior to intervention (e.g., LPI).

\section{$\operatorname{EyeCam}^{\mathrm{TM}}$}

The EyeCam ${ }^{\text {TM }}$ (Clarity Medical Systems, Pleasanton, CA, USA) is a new imaging device derived from the RetCam $^{\mathrm{TM}}$, which was originally designed to photograph the pediatric fundus [30]. With modifications in optical techniques and the inclusion of a wide-field lens, the device can visualize angle structures in a manner similar to direct gonioscopy, but the goniograph produced has a wider view, higher quality, and is less prone to distortions than that produced by traditional goniophotography (Fig. 7).

When EyeCam ${ }^{\mathrm{TM}}$ imaging is performed, the patient is in a supine position in a darkened room and the lens probe is placed on the patient's eye with a coupling solution without direct corneal contact. This minimizes alteration of the angle configuration due to indentation and causes less discomfort than gonioscopy. To better image a particular angle quadrant, the patient is instructed to look in the direction of that angle. The probe is positioned at the limbus opposite the angle being photographed and light from the fiber-optic probe is directed toward the angle of interest and then tilted downward to avoid light affecting the pupil size.

A study compared EyeCam ${ }^{\mathrm{TM}}$ imaging with conventional gonioscopy in 60 eyes with angles ranging from Shaffer grade 0 to 4 on clinical gonioscopy, and 
Fig. 4 Cirrus $^{\mathrm{TM}}$ spectraldomain optical coherence tomography image of open angles. $S C$ Schlemm's canal, $S L$ Schwalbe's line, $T M$ trabecular meshwork, SS scleral spur

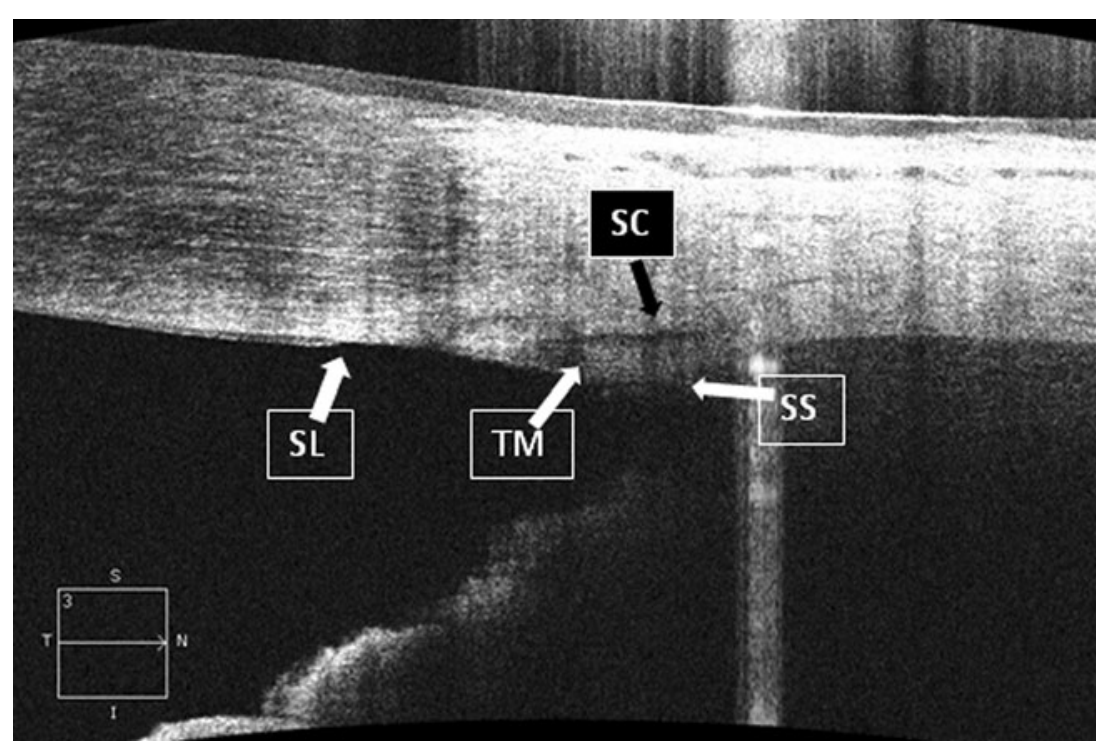

Fig. 5 RTVue ${ }^{\circledR}$ spectraldomain optical coherence tomography image of open angles. SC Schlemm's canal, $S L$ Schwalbe's line, $T M$ trabecular meshwork, SS scleral spur

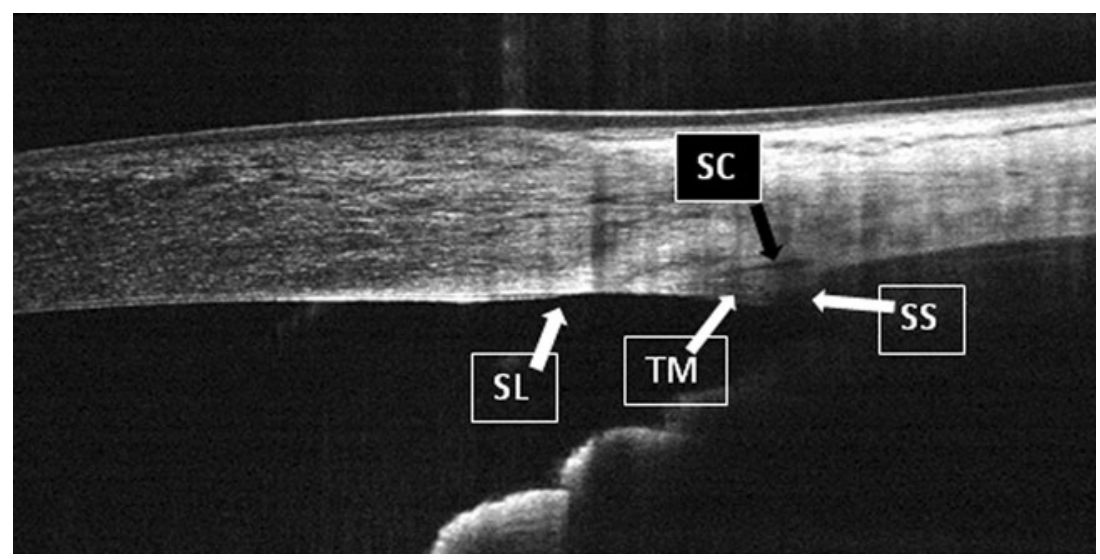

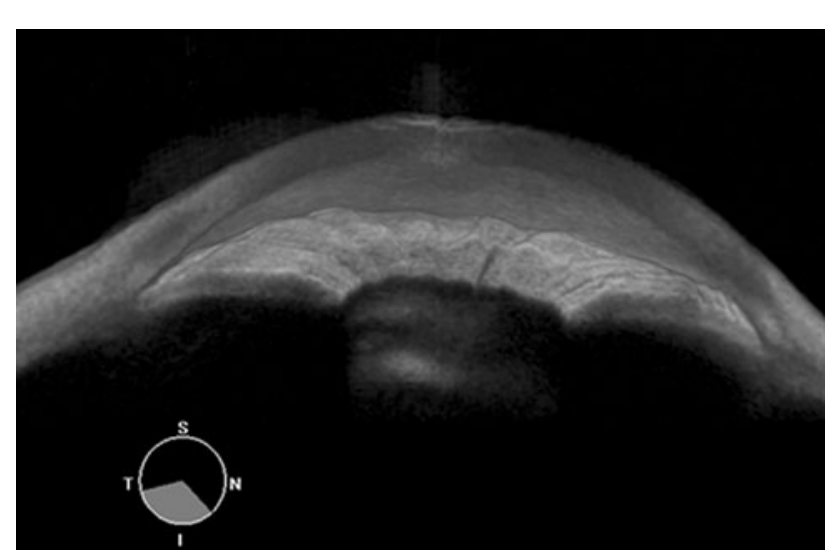

Fig. 6 CASIA $^{\mathrm{TM}}$ swept-source optical coherence tomography scan can rotate $360^{\circ}$ to produce a three-dimensional reconstruction of an eye with a narrow angle

demonstrated that the results from EyeCam ${ }^{\mathrm{TM}}$ imaging are accurate and reliable [31]. Perera et al. [32] found that the agreement between EyeCam ${ }^{\mathrm{TM}}$ imaging and gonioscopy in detecting closed quadrants was good. Baskaran et al. [33] found that the area under the receiver operating characteristic curve (AUC) for detecting eyes with gonioscopic angle closure was similar for goniophotography and EyeCam $^{\mathrm{TM}}$ imaging (AUC 0.93, sensitivity $94.7 \%$, specificity $91.5 \% ; P>0.95)$. The diagnostic performance of EyeCam ${ }^{\mathrm{TM}}$ imaging was better than that of AS-OCT in detecting angle closure when gonioscopic grading was used as the reference standard, and agreement between the two imaging modalities was moderate [34].

This device allows objective comparisons of angle configuration through time, and photographs can be used for patient education. Although the EyeCam ${ }^{\mathrm{TM}}$ is unable to provide quantitative ACA measurements unlike AS-OCT or UBM, it provides a $360^{\circ}$ view of the entire ACA, most similar to gonioscopy itself, which is not possible with other meridional scanners (Fig. 5).

The device has some limitations: ACA imaging takes longer (about 5-10 min per eye) and is more expensive compared with gonioscopy, and more office space is required for supine examination. It is not known if supine positioning widens the angle because of the effect of 


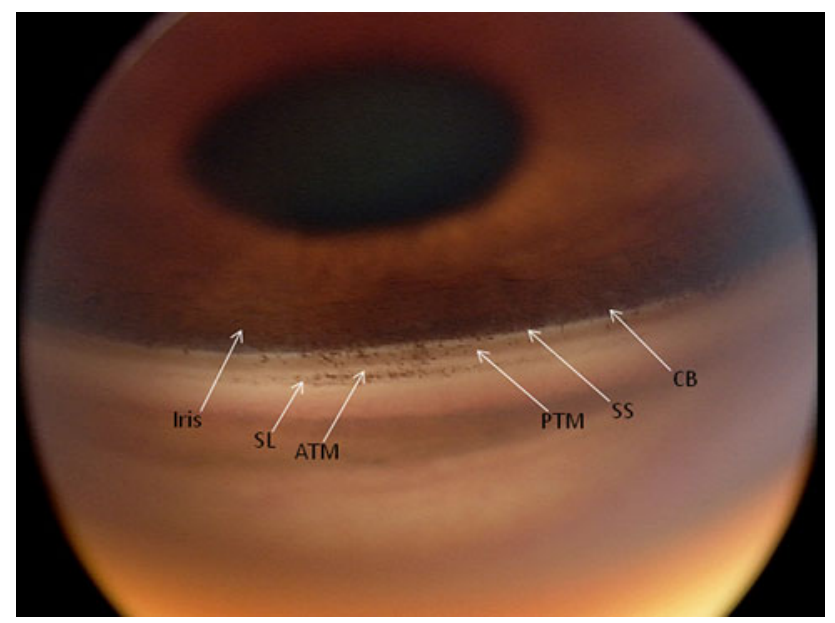

Fig. 7 EyeCam $^{\mathrm{TM}}$ image of open angles. SL Schwalbe's line, ATM anterior trabecular meshwork, PTM posterior trabecular meshwork, $S S$ scleral spur, $C B$ ciliary body

gravity on the lens-iris diaphragm. The device light source delivered via a fiber-optic cable may cause pupil constriction if it is not used properly, artificially widening the ACA. Unlike with dynamic gonioscopy, it is difficult to discern the presence of peripheral anterior synechiae owing to the inability to widen the angle through corneal indentation. Reproducibility may be compromised with further imaging, as each photograph may not be obtained in the exact same location.

\section{Scheimpflug Photography (Pentacam ${ }^{\circledR}$ )}

The Pentacam ${ }^{\circledR}$ (Oculus Optikgeräte, Wetzlar, Germany) is a noncontact slit image photographic device that allows assessment of the anterior segment, from the cornea to the posterior lens. The Pentacam ${ }^{\circledR}$ uses the Scheimpflug principle, which describes the optical properties involved in the photography of objects when their plane is not parallel to the film of the camera. It requires that the plane containing the slit beam and the image plane intersect at one point; with the corresponding angles being equal [35]. The Pentacam ${ }^{\circledR}$ has a rotating Scheimpflug camera that takes up to 50 slit images of the anterior segment in less than $2 \mathrm{~s}$, and software is then used to construct a three-dimensional image. It measures the anterior chamber depth, volume, and angle width in degrees.

With Scheimpflug systems the ACA cannot be directly visualized because the light is unable to penetrate the angle recess and the resolution is insufficient for detailed angle assessment because of light scattering. Therefore, it has limited application in documenting angle closure.
SPAC Scanning Peripheral Anterior Chamber Depth Analyzer

The SPAC scanning peripheral anterior chamber depth analyzer (Takagi Seiko, Nagano-ken, Japan) is a noncontact optical device that indirectly images the angle and does not give detailed information on angle anatomy. Images are then captured by a camera and automatically analyzed by computer. In the slit-lamp-based photographic technique used $\left(60^{\circ}\right.$ offset-similar to the van Herick angle assessment technique), scanning starts at the optical axis toward the periphery and to 21 measurements of anterior chamber depth are obtained. The SPAC scanning peripheral anterior chamber depth analyzer also measures corneal thickness and the radius of corneal curvature in order to derive a more accurate assessment of the anterior chamber depth at various points. The SPAC scan takes $0.67 \mathrm{~s}$ to obtain images captured at $0.4-\mathrm{mm}$ intervals; these are then converted into numerical and categorical grades by comparison with a normative database obtained from a sample of Japanese subjects.

Kashiwagi et al. [36] compared the SPAC scanning peripheral anterior chamber depth analyzer, UBM, and A-scan ultrasonography in detecting changes in the anterior segment of pre-LPI and post-LPI eyes and detected increased peripheral anterior chamber depth after LPI. An increase in angle depth was seen when UBM was used in this population, supporting the SPAC findings. The central anterior chamber depth and axial length did not change when A-scan ultrasonography was used, as has been reported previously.

Several studies indicate that the SPAC findings correlate with angle findings to some extent, but it is unclear if the degree of correlation is high enough for the device to be used effectively for screening for angle closure. More research will be needed to clarify the role that the SPAC scanning peripheral anterior chamber depth analyzer may play in angle assessment [37].

\section{Conclusions}

Dynamic indentation gonioscopy, the present gold standard for angle assessment, is beset by several shortcomings, such as its lack of reproducibility, subjectivity, and operator skill dependence. New methods of angle imaging offer more objectivity, reproducibility, measurement precision, and convenience for both the patient and the observer. Although none of these new methods can replace gonioscopy, the information they provide complements the information obtained from this current reference standard in assessing the ACA. Although these methods have made inroads in attempting to address gonioscopy's drawbacks, 
they are not without their own limitations. With technology rapidly increasing imaging speed and enhancing resolution, especially among AS-OCT ACA devices, it is certain that in the future science will offer more insights into the pathophysiology of angle closure. From this, patients may benefit from improvements in early detection and by way of interventions for angle closure glaucoma.

Disclosure J. M. S. de Leon: none; T. A. Tun: none; S. A. Perera: Payment for lectures including service on speakers bureaus from Carl Zeiss Meditec and Allergan; T. Aung: none.

\section{References}

Papers of particular interest, published recently, have been highlighted as:

- Of importance

1. Foster PJ, Johnson GJ. Glaucoma in China: how big is the problem? Br J Ophthalmol. 2001;85:1277-82.

2. Quigley HA, Broman AT. The number of persons with glaucoma worldwide in 2010 and 2020. Br J Ophthalmol. 2006;90:262-7.

3. Resnikoff S, Pascolini D, Etya'ale D, et al. Global data on visual impairment in the year 2002. Bull World Health Organ. 2004;82:844-51.

4. Sihota R, Lakshmaiah NC, Walia KB, et al. The trabecular meshwork in acute and chronic angle closure glaucoma. Indian $\mathrm{J}$ Ophthalmol. 2001;49:255-60.

5. Ishikawa H, Schuman JS. Anterior segment imaging: ultrasound biomicroscopy. Ophthalmol Clin North Am. 2004;17:7-20.

6. Pavlin CJ, Harasiewicz K, Sherar MD, et al. Clinical use of ultrasound biomicroscopy. Ophthalmology. 1991;98:287-95.

7. Ritch R, Liebmann JM. Role of ultrasound biomicroscopy in the differentiation of block glaucomas. Curr Opin Ophthalmol. 1998;9:39-45.

8. Sbeity Z, Dorairaj SK, Reddy S, et al. Ultrasound biomicroscopy of zonular anatomy in clinically unilateral exfoliation syndrome. Acta Ophthalmol. 2008;86:565-8.

9. Ishikawa H, Uji Y, Emi K. (A new method of quantifying angle measurements based on ultrasound biomicroscopy). Atarashii Ganka. 1995;12:957-60. Japanese.

10. Radhakrishnan S, Goldsmith J, Huang D, et al. Comparison of optical coherence tomography and ultrasound biomicroscopy for detection of narrow anterior chamber angles. Arch Ophthalmol. 2005;123:1053-9.

11. Tello C, Liebmann J, Potash S, et al. Measurement of ultrasound biomicroscopy images: intraobserver and interobserver reliability. Invest Ophthalmol Vis Sci. 1994;35:3549-52.

12. Barkana Y, Dorairaj SK, Gerber Y, et al. Agreement between gonioscopy and ultrasound biomicroscopy in detecting iridotrabecular apposition. Arch Ophthalmol. 2007;125:1331-5.

13. Kaushik S, Jain R, Pandav SS, et al. Evaluation of the anterior chamber angle in Asian Indian eyes by ultrasound biomicroscopy and gonioscopy. Indian J Ophthalmol. 2006;54:159-63.

14. Narayanaswamy A, Vijaya L, Shantha B, et al. Anterior chamber angle assessment using gonioscopy and ultrasound biomicroscopy. Jpn J Ophthalmol. 2004;48:44-9.
15. Goldsmith JA, Li Y, Chalita MR, et al. Anterior chamber width measurement by high-speed optical coherence tomography. Ophthalmology. 2005;112:238-44.

16. Pavlin CJ, Foster FS. Ultrasound biomicroscopy of the eye. New York: Springer; 1994

17. Sakata LM, Lavanya R, Friedman DS, et al. Comparison of gonioscopy and anterior segment ocular coherence tomography in detecting angle closure in different quadrants of the anterior chamber angle. Ophthalmology. 2008;115:769-74.

18. Sakata LM, Lavanya R, Friedman DS, et al. Assessment of the scleral spur in anterior segment optical coherence tomography images. Arch Ophthalmol. 2008;126:181-5.

19. Console JW, Sakata LM, Aung T, et al. Quantitative analysis of anterior segment optical coherence tomography images: the Zhongshan Angle Assessment Program. Br J Ophthalmol. 2008;92:1612-6.

20. Nolan WP, See JL, Chew PT, Friedman DS, Smith SD, Radhakrishnan S, et al. Detection of primary angle closure using anterior segment optical coherence tomography in Asian eyes. Ophthalmology. 2007;114:33-9.

21. Dada T, Sihota R, Gadia R, et al. Comparison of anterior segment optical coherence tomography and ultrasound biomicroscopy for assessment of the anterior segment. J Cataract Refract Surg. 2007;33:837-40.

22. Wojtkowski M, Bajraszewski T, Gorczy pska I, et al. Ophthalmic imaging by spectral optical coherence tomography. Am J Ophthalmol. 2004;138:412-9.

23. Quek DT, Narayanaswamy AK, Tun TA, et al. Comparison of two spectral domain optical coherence tomography devices for angle closure assessment. Invest Ophthalmol Vis Sci. 2012;53:5131-6.

24. Wylegala E, Teper S, Nowiñska AK, et al. Anterior segment imaging: fourier-domain optical coherence tomography versus time-domain optical coherence tomography. J Cataract Refract Surg. 2009;35:1410-4.

25. Wong HT, Lim MC, Sakata LM, et al. High-definition optical coherence tomography imaging of the iridocorneal angle of the eye. Arch Ophthalmol. 2009;127:256-60.

26. Yun S, Tearney G, de Boer J, et al. Highspeed optical frequencydomain imaging. Opt Express. 2003;11:2953-63.

27. Yasuno Y, Madjarova VD, Makita S, et al. Three-dimensional and high-speed swept-source optical coherence tomography for in vivo investigation of human anterior eye segments. Opt Express. 2005;13(26):10652-64.

28. Liu S, Yu M, Cong Ye, et al. Anterior chamber angle imaging with swept-source OCT: an investigation on variability of angle measurement. Invest Ophthalmol Vis Sci. 2011;52:8598-603.

29. - Ho SW, Baskaran M, Zheng C, et al. Swept source optical coherence tomography measurement of the iris-trabecular contact (ITC) index: a new parameter for angle closure. Graefes Arch Clin Exp Ophthalmol. 2012. doi:10.1007/s00417-012-2158-6. SS-OCT $\left(C A S I A^{T M}\right)$ provides, for the first time, an estimation of angle closure which is more analogous to that derived from gonioscopy, and this study showed that interobserver and intraobserver agreement for this objective parameter of extent of angle closure (iridotrabecular contact index) was good.

30. Erraguntla V, MacKeen LD, Atenafu E, et al. Assessment of change of optic nerve head cupping in pediatric glaucoma using the RetCam 120. J AAPOS. 2006;10:528-33.

31. Calafati J, Naqi A, Ahmed I. Digital imaging system an alternative to traditional process, US edn. Thorofare: Ocular Surgery News; 2009.

32. Perera SA, Baskaran M, Friedman DS, et al. Use of EyeCam for imaging the anterior chamber angle. Invest Ophthalmol Vis Sci. 2010;51:2993-7. 
33. Baskaran M, Perera SA, Nongpiur ME, et al. Angle assessment by EyeCam, goniophotography, and gonioscopy. J Glaucoma. 2012;21:493-7.

34. Baskaran M, Aung T, Friedman DS, et al. Comparison of EyeCam and anterior segment optical coherence tomography in detecting angle closure. Acta Ophthalmol. 2012;90(8):e621-5.

35. Masters BR. Three-dimensional microscopic tomographic imaging of the cataract in a human lens in vivo. Opt Express. 1998;3:332-8.
36. Kashiwagi K, Abe K, Tsukahara S. Quantitative evaluation of changes in anterior segment biometry by peripheral laser iridotomy using newly developed scanning peripheral anterior chamber depth analyser. Br J Ophthalmol. 2004;88:1036-41.

37. Friedman DS, He M. Anterior chamber angle assessment techniques. Surv Opthalmol. 2008;53:250-73. 